\title{
Sphingosine-1-phosphate mobilizes osteoclast precursors and regulates bone homeostasis
}

\author{
Masaru Ishii ${ }^{1,3,4}$, Jackson G. Egen ${ }^{1}$, Frederick Klauschen ${ }^{2}$, Martin Meier-Schellersheim², \\ Yukihiko Saeki ${ }^{4}$, Jean Vacher ${ }^{5}$, Richard L. Proia ${ }^{6}$, and Ronald N. Germain. ${ }^{1,2}$ \\ ${ }^{1}$ Lymphocyte Biology Section, Laboratory of Immunology, National Institutes of Health, Bethesda, \\ MD 20892-1892, USA \\ 2Program in Systems Immunology and Infectious Disease Modeling, National Institute of Allergy \\ and Infectious Diseases, National Institutes of Health, Bethesda, MD 20892-1892, USA \\ ${ }^{3}$ Laboratory of Biological Imaging, WPI-Immunology Frontier Research Center, Osaka University, \\ Osaka 565-0871, Japan \\ ${ }^{4}$ Department of Clinical Research, National Osaka Minami Medical Center, Osaka 586-8521, Japan \\ 5Institut de Recherches Cliniques de Montreal, Quebec H2W 1R7, Canada \\ ${ }^{6}$ Genetics of Development and Disease Branch, National Institute of Diabetes and Digestive and \\ Kidney Diseases, National Institutes of Health, Bethesda, MD 20892-1892, USA
}

\begin{abstract}
Osteoclasts (OCs) are bone-resorbing multinuclear giant cells that differentiate from mononuclear macrophage/monocyte-lineage hematopoietic precursors. Although many molecules are known to contribute to OC differentiation, RANKL chief among them, the mechanisms controlling the recruitment and homing of $\mathrm{OC}$ precursors (OPs) to the bone surface have not been elucidated. Here we report that sphingosine-1-phosphate (S1P) controls the movement of OPs between the blood and their site of differentiation. Cells with the properties of OPs express functional S1P $\mathrm{P}_{1}$ receptors and exhibit positive chemotaxis along an S1P gradient in vitro. Intravital two-photon imaging of bone tissues revealed that a potent $\mathrm{S}_{1} \mathrm{P}_{1}$ agonist, SEW2871, stimulated motility of OP-containing monocytoid populations in vivo. $\mathrm{OC} /$ monocyte $(\mathrm{CD} 11 \mathrm{~b})$ lineage-specific conditional $\mathrm{S}_{1} \mathrm{P}_{1}$ knockout mice showed osteoporotic changes due to increased OC attachment to bone surface, suggesting a crucial role of the S1P-S1P 1 system in recirculation of OPs to blood where S1P levels are high. Furthermore, treatment with the $\mathrm{S}_{1} \mathrm{P}_{1}$ agonist FTY720 relieved ovariectomy-induced osteoporosis in mice by facilitating recirculation of OP-containing cell populations and reducing the number of mature OCs attached to the bone surface. This study provides evidence that S1P controls the migratory behavior of OPs, dynamically regulating bone mineral homeostasis, and identifies a critical control point in osteoclastogenesis that may be promising as a therapeutic target.
\end{abstract}

Osteoclasts (OCs) are the only somatic cells with bone-resorbing capacityand as such, they play a critical role not only in normal bone homeostasis (called "bone remodeling") but also in the pathogenesis of bone destructive disorders such rheumatoid arthritis and

Correspondence should be addressed to R.N.G. (rgermain@niaid.nih.gov).

Author Contributions M.I. performed most of experiments, with the assistance of J.G.E. and Y.S. for two-photon microscopy and for the in vitro osteoclast culture system, respectively. F.K. developed the unsupervised segmentation software and performed the computational analyses used to quantify the osteoclast-bone surface interface, with the assistance of M. M-S. J.V. and R.L.P. generated CD11b-Cre transgenic and S1P1 loxP knock-in mice, respectively. R.N.G. helped M.I. in designing and interpreting experiments, as well as in writing the paper. 
osteoporosis $^{1}$. A major focus of research in the field has been on gene regulation by osteoclastogenic cytokines, such as receptor activator of NFאB - ligand (RANKL) and $\mathrm{TNF} \alpha$, both of which have been well documented to contribute to osteoclast terminal differentiation ${ }^{2,3}$. A critical process that has been less well studied is the trafficking of OC precursors (OPs) to and from the bone surface, where they undergo cell fusion to form the fully differentiated multinucleated cells that mediate bone resorption. Do the same cytokines (RANKL and TNF $\alpha$ ) known to be critical for mature OC generation also control this initial stage of osteoclastogenesis or is another set of molecules involved? To investigate the factors involved in regulation of OP cell dynamics at the bone surface, we examined the likely role of chemokines and screened several such molecules for their capacity to affect migration of monocytes with the properties of OP. Here we report that sphingosine-1-phosphate, a lipid mediator enriched in blood ${ }^{4,5}$, induces chemotaxis and regulates migration of OPs not only in culture but in vivo, contributing to dynamic control of bone mineral homeostasis. We also show that pharmacologic manipulation of the S1P signaling pathway in monocytoid cells containing OPs has a dramatic effect on bone mineralization, suggesting that this pathway may be of interest as a drug target in diseases in which OC over- or under-activity underlies pathology.

Because of its role in controlling cell migration in other tissues, we initially focused on S1P and the Edg family of receptors (collectively known as S1P receptors) responsive to this lipid mediator. RAW264.7, a murine monocyte cell line, and mouse bone marrow-derived, M-CSFdependent monocytes (BM-MDM), both of which can differentiate into osteoclast-like cells upon exposure to RANKL and represent widely employed in vitro models of OPs, each express mRNAs encoding two receptors for $\mathrm{S} 1 \mathrm{P}\left(\mathrm{S}_{1} \mathrm{P}_{1}\right.$ and $\left.\mathrm{S}_{1} \mathrm{P}_{2}\right)$. The expression of $\mathrm{S}_{1} \mathrm{P}_{1}$ mRNA decreased upon RANKL stimulation (Fig. 1a, b), and this transcriptional repression was dependent on NF- $\mathrm{kB}$ (blocked by BAY11-7085), but not on NF-AT (blocked by cyclosporin A) (Fig. 1b), both of which have been recognized as crucial transcriptional factors for osteoclastogenesis ${ }^{1-3} . \mathrm{S} \mathrm{P}_{1}$ protein expression, as detected by immunocytochemistry, was also reduced by RANKL stimulation (Fig. 1c). In accord with these in vitro findings, strong immunoreactivity for $\mathrm{S}_{1} \mathrm{P}_{1}$ could be detected in mononuclear cells in native bone tissues (Fig. 1d, arrowheads), whereas weaker staining was seen in large cells lining bone trabeculae (Fig. $1 \mathrm{~d}$, asterisks) that are likely to be mature OCs (Mean fluorescence intensities; $49.1 \pm 5.8$ and $19.4 \pm 2.0$, in mononuclear cells and attached cells, respectively $(\mathrm{p}=0.0003))$. Both RAW264.7 (Fig. 1f) and BM-MDM (not shown) exhibited positive chemotactic responses to an S1P gradient in vitro and these responses were greatly attenuated by pretreatment with RANKL (Fig. 1f). This latter finding is concordant with the results showing RANKL-dependent reduction in the expression of $\mathrm{S}_{1} \mathrm{P}_{1}$ (Fig. 1a). S1P-directed chemotaxis was blocked by pertussis toxin (PTX) ${ }^{6}$ and NSC23766 ${ }^{7}$ (Fig. 1f) and S1P induced an increase in the GTPbound, active form of Rac (GTP-Rac) (Fig. 1e), suggesting the involvement of $\mathrm{G}_{\mathrm{i}} \alpha$ and Rac in the $\mathrm{S}_{1} \mathrm{P}_{1}$ chemotactic signaling pathway in these cells, in accordance with previous reports 8 , 9. In vitro chemotaxis of RAW264.7 was also examined using a dynamic imaging approach, where cells could be observed vigorously migrating toward S1P-containing medium (Fig. 1g; Supplementary Videos 1 and 2). We also detected S1P-induced up-regulation of integrins expressed in monocytoid cells, suggesting that S1P-mediated alterations in cell adhesion could contribute to chemotaxis in vivo (Supplementary Fig. 1).

To investigate whether OP migration is affected by S1P gradients in vivo, we performed intravital two-photon imaging of calvaria bone tissues ${ }^{10,11}$ and examined the migratory behavior of monocytoid cells containing OPs resident in the marrow spaces. We used two strains of mice expressing EGFP in subsets of myeloid cells: $\mathrm{CX}_{3}$ CR1-EGFP knock-in (heterozygous) mice ${ }^{12,13}$ and CSF1R-EGFP transgenic mice ${ }^{14}$. In both strains, hematopoietic monocyte-lineage cell types predominantly expressed EGFP ${ }^{12,13}$ and we confirmed that tartrate-resistant acid phosphatase (TRAP)-positive mature OCs expressed EGFP in these animals (Supplementary Fig. 2), suggesting that $\mathrm{EGFP}^{+}$cells do contain OP monocytes. Nearly 
two thirds of $\mathrm{CX}_{3} \mathrm{CR} 1-\mathrm{EGFP}$ cells and half of CSF1R-EGFP cells express RANK, a receptor for RANKL (Supplementary Table 1), suggesting a capacity for many of the fluorescent cells to differentiate into mature OCs.

$\mathrm{CX}_{3} \mathrm{CR} 1-\mathrm{EGFP}$ positive cells present in bone marrow stromal locations or at the bone surface and not in the blood sinusoidal spaces were generally stationary under control conditions (Fig. 2a, upper panels; Supplementary Video 3). A subset of the labeled cells became motile shortly after the intravenous application of SEW2871 $15,16(5 \mathrm{mg} / \mathrm{kg})$, a selective agonist for the $\mathrm{S} 1 \mathrm{P}_{1}$ receptor (Fig. 2a, lower panels; Supplementary Video 4), with some of the mobilized cells entering the blood circulation (not shown). A similar but less pronounced effect of SEW2871 on myeloid cell motility was observed in the bone marrow of CSF1R-EGFP transgenic mice (Supplementary Fig. 3; Supplementary Videos 5 and 6). The difference in the fraction of cells mobilized by SEW2871 treatment in the two mouse strains is likely due to the fact that the EGFP-expressing subsets in these animals are not completely overlapping (Supplementary Table 1). Consistent with these findings, we also observed an elevated percent and absolute number of monocytoid cells in peripheral blood and their reciprocal decrease in bone marrow, but not spleen or liver, following SEW2971 treatment as compared to vehicle controls (Supplementary Fig. 4 and Table 2). These results suggest that bone marrow-resident monocytes, including many OPs, become motile and exit marrow spaces in response to S1P stimulation.

To evaluate the in vivo impact of S1P-directed chemotaxis of OPs on bone homeostasis, we examined mice deficient in $\mathrm{S}_{1} \mathrm{P}_{1}$. Because $\mathrm{S} 1 \mathrm{P}_{1}$ deficiency causes embryonic lethality ${ }^{17}$, we generated - osteoclast/monocyte-specific $\mathrm{S}_{1} \mathrm{P}_{1}$-deficient $\left(\mathrm{cS} 1 \mathrm{P}_{1}^{-/-}\right)$mice by crossing animals bearing conditional $\mathrm{S}_{1} \mathrm{P}_{1}$ knockout alleles $\left(\mathrm{S}_{1} \mathrm{P}_{1}{ }^{\text {loxP }}\right)^{18}$ to transgenic mice expressing Cre under the CD11b promoter ${ }^{19}$, which facilitates deletion of loxP-flanked sequences in monocyte-lineage myeloid cells including OCs. S1P 1 mRNA was essentially undetectable by conventional and real-time quantitative RT-PCR analyses in CD11 $\mathrm{b}^{+}$myeloid cells purified from the $\mathrm{cS}_{1} \mathrm{P}_{1}^{-/-}$mice (Supplementary Fig. 5a, b) and immunoreactivity for $\mathrm{S}_{1} \mathrm{P}_{1}$ was greatly diminished in ${\mathrm{cS} 1 \mathrm{P}_{1}}^{-/-}$bone tissues (Supplementary Fig. 5c), indicating almost complete ablation of $\mathrm{S}_{1} \mathrm{P}_{1}$ expression in this lineage.

Morphohistometric analyses using $\mu \mathrm{CT}$ showed that femora of mice genotyped as $\mathrm{S} 1 \mathrm{P}_{1}$ loxP/loxP $\mathrm{CD} 11 \mathrm{~b}-\mathrm{Cre}\left(\mathrm{cS} \mathrm{P}_{1}^{-/-}\right)$were osteoporotic, compared with those of control $\left(\mathrm{S}_{\mathrm{P}}{ }_{1}^{+/+} \mathrm{CD} 11 \mathrm{~b}-\mathrm{Cre}\right.$ ) littermates (Fig. 3a, upper panels). Bone tissue density (= BV / TV) of $\mathrm{cS}_{1} \mathrm{P}_{1}^{-/-}$mice was significantly lower than that of controls (Fig. 3b), and both trabecular thickness (Tb.Th.) and trabecular density (Tb.N.) were decreased in $\mathrm{cS}_{1} \mathrm{P}_{1}{ }^{-/}$bones (Fig. 3c). Mice genotyped as $\mathrm{S} 1 \mathrm{P}_{1}$ loxP/+ $\mathrm{CD} 11 \mathrm{~b}-\mathrm{Cre}$ (heterozygous) were indistinguishable from controls, indicating that a single $\mathrm{S}_{1} \mathrm{P}_{1}$ allele is sufficient to sustain normal $\mathrm{S}_{1} \mathrm{P}_{1}$-mediated functions.

Bone surface attachment of mature OCs was evaluated by immunohistological examination. Both OCs and bone matrices were visualized simultaneously by fluorescence-based staining for TRAP ${ }^{20}$ and by collagen-derived second harmonic signals elicited by two-photon excitation $^{21}$, respectively (Supplementary Fig. 6). Images were automatically segmented offline using a custom software package (F.K., submitted). An "osteoclast attachment ratio" (= bone surface occupied by osteoclasts / total bone surface) was calculated automatically without manual intervention (Fig. 3a, lower panels, and Supplementary Fig. 6). The computational analyses showed that osteoclast attachment to the bone surface was significantly enhanced in $\mathrm{cS}_{1} \mathrm{P}_{1}^{-/-}$animals, as compared to controls (Fig. 3d), consistent with - the decrease in bone density in $\mathrm{cS} 1 \mathrm{P}_{1}{ }^{-/-}$mice (Fig. 3b, c). Because S1P has no measurable effect on in vitro osteoclast differentiation stimulated by RANKL (Supplementary Fig. 7), a reasonable interpretation of 
these data is that the loss of $\mathrm{S}_{1} \mathrm{P}_{1}$ function alters the chemotactic behavior of OPs rather than their terminal maturation to OCs or the bone-resorbing action of OCs.

Because the concentration of S1P in blood is higher than that in tissues, S1P-mediated chemotaxis of osteoclast precursors thus would be expected to contribute to their recirculation from bone tissues to systemic blood flow, limiting formation of mature osteoclasts and reducing bone destruction. The present result showing the increased deposition of $\mathrm{S}_{1} \mathrm{P}_{1}^{-/-}$OCs on the bone surface strongly supports the concept of S1P-driven precursor recirculation. It has already been reported that OP monocytes (RAW264.7) show positive chemotaxis in vitro toward CXCL12 (SDF-1), a chemokine secreted from bone marrow stromal cells ${ }^{22}$, leading to a straightforward competitive model for the migration and positioning of OCs (Supplementary Fig. 8): Monocyte-lineage OC precursors come close to the bone surface attracted by CXCL12, however not all of the recruited cells undergo differentiation, and some of the precursors recirculate into the blood flow due to attraction by S1P. Some OPs may be directly derived within bone marrow spaces (Supplementary Fig. 8, dashed arrow), and they are also susceptible to S1P-driven "circulation" into the systemic flow. During OC differentiation induced by RANKL, the expression of $\mathrm{S}_{1} \mathrm{P}_{1}$ is repressed (Fig. 1a), which ensures stable localization of the maturing cells on the bone surface. Regulation of OC generation by competing chemokine pathways that attract precursors to and from the bone surface allows finely-tuned dynamic control of osteoclastogenesis during health and in various conditions such as inflammation and metabolic disorders. Bone surface CXCL12 expression has recently been shown to be regulated by sympathetic neuron stimulation ${ }^{23}$, suggesting an interesting interplay between circulating S1P and the autonomic nervous system for the regulation of OP mobilization.

This newly revealed role of S1P-directed recirculation of OP in bone homeostasis prompted us to examine possible therapeutic implications of this regulatory pathway. FTY720

(Fingolimod) is metabolized after administration to anagonist for four of the five S1P receptors, including $\mathrm{S}_{1} \mathrm{P}_{1}$ (but not $\mathrm{S}_{2} \mathrm{P}_{2}$ ), and has emerged as a clinically promising novel immunosuppressive drug 24,25 that presumably acts by limiting effector lymphocyte egress from lymph nodes. FTY720 is currently being tested clinically in multiple sclerosis and allogeneic transplantation ${ }^{26,27}$, but has not been examined for its effect on bone homeostasis. Here we show that FTY720 has a capacity to ameliorate bone loss in a model of postmenopausal osteoporosis.

Either ovariectomized or sham-operated mice were administered FTY720 (3 mg / kg / day) for four weeks after which excised femora were analyzed by $\mu \mathrm{CT}$ (Fig. 4a, b). FTY720 treatment significantly prevented bone density loss after ovariectomy, but had no effects under control (sham-operated) conditions. Computational morphometric analyses showed that OC attachment was increased upon ovariectomy and partially restored to normal levels by FTY720 administration. We confirmed that FTY720 had no effect on estrogen levels after ovariectomy (Supplementary Table 3). These results suggest that FTY720 relieves ovariectomy-induced bone loss by reducing OC deposition onto bone surfaces. This effect correlated with an increase in circulating $\mathrm{CX}_{3} \mathrm{CR} 1-\mathrm{EGFP}^{+} \mathrm{F} 4 / 80^{+}$(Fig. $4 \mathrm{c}$ ) and CD11b ${ }^{+}$monocytes (Supplementary Fig. 9), both of which populations include OPs, and a concomitant decrease in monocytoid cells including OPs in the bone marrow (Supplementary Fig. 9 and Table 2). In addition, intravital two-photon imaging revealed that FTY720 significantly stimulated motility of $\mathrm{CX}_{3} \mathrm{CR} 1$ EGFP $^{+}$monocyte-lineage cells (Fig. $4 \mathrm{~d}$ and Supplementary Video 7). The short-term mobility change seen using FTY720 (as observed by intravital microscopy) was less prominent than that obtained with SEW2871 (Fig. 2). This difference may arise because SEW2871 is active upon intravenous injection, whereas FTY720 needs to be metabolized (phosphorylated) before becoming functional (Supplementary Fig. 10). We cannot rule out, however, that phosphoFTY720 can activate $\mathrm{S} 1 \mathrm{P}$ receptors other than $\mathrm{S}_{1} \mathrm{P}_{1}$ that are expressed on other cell types, such as endothelial cells. FTY720 was shown to have no effects on the expression of RANKL or 
osteoprotegerin (not shown). Taken together, these results indicate that FTY720, a potent $\mathrm{S}_{1} \mathrm{P}_{1}$ agonist, promotes the recirculation of OP monocytes from bone surface, an effect that counteracts ovariectomy-induced augmentation of osteoclastogenesis. This agonist effect of FTY720 on myeloid cells directly accessible to the blood circulation differs from the

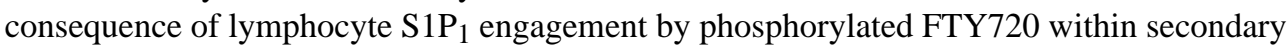
lymphoid tissues, in which case downmodulation of the receptor results in functional antagonism of this pathway.

In conclusion, the present study provides evidence that $\mathrm{S} 1 \mathrm{P}$, as a circulation-attraction molecule, controls the migratory behavior of OPs, dynamically regulating bone mineral homeostasis in collaboration with bone-attraction molecules, such as CXCL12, and identifies a critical control point in osteoclastogenesis that may be promising as a therapeutic target.

\section{Methods summary}

RAW264.7, a murine macrophage/monocyte lineage cell line, and mouse bone marrowderived M-CSF-dependent monocytes (BM-MDM), containing osteoclast precursor cells, were cultured and stimulated to differentiate using RANKL as previously described ${ }^{28,29}$. In vitro chemotactic activity was evaluated both using a modified Boyden's chamber ${ }^{30}$ or with a visually-accessible chemotactic chamber, EZ-Taxiscan. In vivo S1P-directed chemotaxis of OP monocytes was visualized by intravital two-photon microscopy of mouse calvaria bone tissues of heterozygous $\mathrm{CX}_{3}$ CR1-EGFP knock-in mice ${ }^{12}$ and of CSF1R-EGFP transgenic ${ }^{14}$ mice, according to a protocol modified from a previous report ${ }^{10,11}$. The generation of the loxPflanked $S 1 P_{1}$ allele $\left(S 1 P_{l}{ }^{l o x P}\right)^{18}$ and osteoclast/monocyte lineage-specific $C D 11 b$-Cre transgenic mice ${ }^{19}$ have been described previously. Histomorphometry of femurs from $\mathrm{OC} /$ monocyte-lineage specific $\mathrm{S}_{1} \mathrm{P}_{1}$ conditional knockout $\left(\mathrm{cS}_{1} \mathrm{P}_{1}{ }^{--}\right)$mice, as well as mice ovariectomized / sham-operated and treated with FTY720 or vehicle, were performed using $\mu \mathrm{CT}$. Assessment of osteoclast attachment to the bone surface was performed using a newly developed fully automated segmentation approach to analyze two-photon images of bone tissue sections in which OCs and bone trabeculae were visualized with fluorescent TRAP staining $^{20}$ and 2 nd harmonic generation from collagen fibers ${ }^{21}$, respectively. To examine the composition of peripheral blood mononuclear cells (PMCs), the cells were stained with fluorophore-conjugated anti-F4/80, anti-CD11b, and anti-CD3 using conventional methods. Flow cytometric data were collected on a FACSCalibur and analyzed with FlowJo software. All mice were bred and maintained under specific pathogen-free conditions at the animal facility of NIH, according to NIH institutional guidelines under an approved protocol. For statistical analyses, the Mann-Whitney rank sum test was used to calculate $p$ values for highly skewed distributions. For Gaussian-like distributions, two-tailed $t$-tests were used.

\section{Methods}

\section{Cell culture}

RAW264.7, a murine macrophage/monocyte lineage cell line, and mouse bone marrowderived M-CSF-dependent monocytes (BM-MDM), containing osteoclast precursor cells, were cultured as previously described ${ }^{28,29}$. To stimulate osteoclastogenesis, $50 \mathrm{ng} / \mathrm{ml}$ RANKL (PeproTech) was added to the medium and the cells were incubated for 3 to 4 days.

\section{Conventional and quantitative (real-time) RT-PCR amplification}

Conventional RT-PCR was performed as described previously ${ }^{29}$. Primers used are listed in Supplementary Table 4. Relative quantification with real-time RT-PCR was performed using an ABI PRISM 7900 (Applied Biosystems Inc.) with an Assay-on Demand TaqMan probe and relevant primers (S1P 1 , Assay ID \#Mm00514644_m1; CD11b/integrin $\alpha_{\mathrm{M}}$, Assay ID 
\#Mm01271259_g1; CD18/integrin $\beta_{2}$, Assay ID \#Mm00434513_m1; GAPDH, Assay ID

\#Mm99999915_g1), according to the manufacturer's instructions.

\section{Determination of Rac activity}

Pulldown assay methods to determine the GTP-bound active form of Rac have been described in detail previously ${ }^{30}$.

\section{In vitro chemotaxis chamber assay}

Chemotactic migration of cells was measured in a modified Boyden chamber as described previously ${ }^{27}$.

\section{EZ-Taxiscan chemotaxis assay}

Chemotaxis experiments were also conducted in an EZ-Taxiscan chamber according to the manufacturer's protocol (Effector Cell Institute). The EZ-Taxiscan is a visually-accessible chemotactic chamber, in which one compartment containing ligand (S1P) and another compartment containing cells are connected by a microchannel. Phase contrast images of migrating cells were acquired at 1 minute interval.

Mice

C57BL/6 mice, $\mathrm{CX}_{3}$ CR1-EGFP knock-in mice ${ }^{12}$, CSF1R (M-CSF receptor)-EGFP transgenic mice ${ }^{14}$, were obtained from The Jackson Laboratory. The generation of the loxP-flanked $S 1 P_{1}$ allele $\left(S 1 P_{l}^{l o x P}\right)^{18}$ and osteoclast/monocyte lineage-specific $C D 11 b$-Cre transgenic mice ${ }^{19}$ were described previously. $S 1 P_{1}^{+/+} \times C D 11$ b-Cre and $S 1 P_{1}^{l o x P /+} x C D 11 b-C r e$ littermates were born at expected frequencies and were viable and fertile, but live

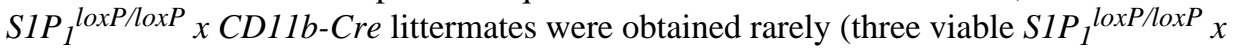
$C D 11 b$-Cre out of 59 total mice), suggesting possible embryonic lethality as seen with global $\mathrm{S}_{1} \mathrm{P}_{1}$ deficiency. All mice were bred and maintained under specific pathogen-free conditions at animal facilities of NIH. Mutant mice were genotyped by PCR ${ }^{18,19}$. All mice were housed and handled according to NIH institutional guidelines under an approved protocol.

\section{Immunohistochemistry}

Immunohistological analyses were performed as described previously ${ }^{2}$. Anti-mouse $\mathrm{S}_{1} \mathrm{P}_{1}$ rabbit polyclonal antibody was purchased from Affinity Bioreagents. In some experiments, fluorescence-based staining for TRAP ${ }^{20}$ with ELF 97 substrate (Molecular Probes) was used with some modifications.

\section{Two-photon intravital bone tissue imaging}

Intravital microscopy of mouse calvaria bone tissues was performed using a protocol modified from a previous report ${ }^{10,11}$. Mice were anesthetized with isoflurane (Baxter, $2.5 \%$ vaporized in an 80:20 mixture of $\mathrm{O}_{2}$ and air), and the hair in the neck and scalp was removed with hair removal lotion (Nair). The frontoparietal skull was exposed and the mouse head was immobilized in a custom-made stereotactic holder. A catheter was placed into the tail vein with a 30-gauge needle attached to PE-10 tubing (Becton Dickinson). The imaging system was composed of an LSM510 NLO multiphoton microscope (Carl Zeiss) driven by a Chameleon XR Ti:Sapphire laser (Coherent) tuned to $880 \mathrm{~nm}$, and an inverted microscope (Axiovert 200; Carl Zeiss) equipped with a 40X water immersion objective (Achroplan IR, N.A. 0.8; Carl Zeiss). Fluorescent cells were detected through a bandpass emission filter at $525 / 50 \mathrm{~nm}$ (for EGFP). Vessels were visualized by injecting $70 \mathrm{kDa}$ Texas Red conjugated-dextran (detected using a $620 / 60 \mathrm{~nm}$ filter) i.v. immediately prior to imaging. Image stacks were collected at 3 $\mu \mathrm{m}$ vertical step size at a depth of $100-150 \mu \mathrm{m}$ below the skull bone surface. For $3 \mathrm{D}$ videos, 4 
sequential image stacks were acquired at $3 \mu \mathrm{m} \mathrm{z}$ spacing to cover a volume of $154 \mu \mathrm{m} \times 154$ $\mu \mathrm{m} \times 9.0 \mu \mathrm{m}$. The time resolution was 1 minute. Raw imaging data were processed with Imaris (Bitplane) with a Gaussian filter for noise reduction.

\section{Ovariectomy and FTY720 treatment}

Twelve 12-week-old female, ovariectomized or sham-operated, C57BL/6J mice were purchased from Charles River Laboratories. Mice were injected i.p. either with FTY720 (3 mg/ $\mathrm{kg}$, Cayman Chemical) dissolved in a vehicle (PBS containing 5\% acidified DMSO and 30\% fatty acid-free BSA) or with vehicle only, daily for four weeks. The mice were then sacrificed and femurs were excised and fixed. Uteri of all the animals were excised and were weighed to evaluate the effect of ovariectomy.

\section{Bone histomorphometry}

Trabecular bone morphometry within the metaphyseal region of distal femur was quantified using micro-CT ( $\mu \mathrm{CT} 40$, Scanco Medical AG). Volumeric regions for trabecular analysis were selected within the endosteal borders to include the central $80 \%$ of vertebral height and secondary spongiosa of femoral metaphyses located $\sim 6 \%$ of the total length from the growth plate. Trabecular morphometry was characterized by measuring the bone volume fraction (bone volume / total volume, BV/TV), trabecular thickness (Tb. Th) and trabecular number (Tb. N.) To assess the attachment of osteoclasts to the bone surface, osteoclasts and bone trabeculae were fluorescently visualized in sections by two-photon microscopy. In order to identify osteoclasts, fluorescence based TRAP staining ${ }^{20}$ was performed. Collagen-enriched bone matrices could be visualized using the 2 nd harmonic emission from collagen fibers excited by infrared lasers. Sections were examined using a two-photon laser microscope (laser was tuned to $780 \mathrm{~nm}$ ). Fluorescence was detected through bandpass emission filters at 370-450 $\mathrm{nm}$ (for $2 \mathrm{nd}$ harmonic emission from bone matrices) or 525/50 $\mathrm{nm}$ (for TRAP staining). Image analysis was performed using a fully automated segmentation approach (no manual tracing of cell/tissue boundaries or other user intervention required) and the "osteoclast attachment ratio" was calculated as the ratio "bone surface attached by osteoclasts / total bone surface" (F.K. submitted).

\section{Flow cytometry}

All reagents were purchased from BD PharMingen. To examine the composition of peripheral blood mononuclear cells (PMC), blood was collected from the retro-orbital plexus with a heparinized glass pipette, from mice treated intraperitoneally 4 hours previously with FTY720 $(3 \mathrm{mg} / \mathrm{kg}$ ) or vehicle (Fig. 4), or treated intravenously 1 hour previously with SEW2871 (5 mg/ $\mathrm{kg}$ ) or vehicle (Supplementary Fig. 4). After removing the red blood cells by ACK lysis buffer (Invitrogen), cells were stained with Alexa647-conjugated anti-F4/80 (Fig. 4), or FITCconjugated anti-CD11b and PE-Cy7-conjugated anti-CD3 (Supplementary Figs. 4 and 9), using conventional methods. Flow cytometric data were collected on a FACSCalibur (Becton Dickinson) and analyzed with FlowJo software (TreeStar).

\section{Supplementary Material}

Refer to Web version on PubMed Central for supplementary material.

\section{Acknowledgments}

We thank Drs. Ulrich H. von Andrian and Irina B. Mazo (Department of Pathology, Harvard Medical School) for their help with the technique of intravital skull bone imaging. We also thank Drs. Yoh Takuwa and Naotoshi Sugimoto (Department of Physiology, Kanazawa University of Medicine, Japan) for helpful discussions, and Drs. Philip M. Murphy and Sundararajan Venkatesan (NIAID, NIH) for their help in imaging in vitro chemotaxis using the EZTaxiscan. This work was supported in part by the Intramural Research Program of the NIAID, NIH, U.S. Department 
of Health and Human Services, and by a fellowship grant to M. I. from the International Human Frontier Science Program.

\section{References}

1. Teitelbaum SL. Bone resorption by osteoclasts. Science 2000;289:1504-1508. [PubMed: 10968780]

2. Teitelbaum SL, Ross FP. Genetic regulation of osteoclast development and function. Nat Rev Genet 2003;4:638-649. [PubMed: 12897775]

3. Karsenty G, Wagner EF. Reaching a genetic and molecular understanding of skeletal development. Dev Cell 2002;2:389-406. [PubMed: 11970890]

4. Rosen H, Goetzl EJ. Sphingosine 1-phosphate and its receptors: an autocrine and paracrine network. Nat Rev Immunol 2005;5:560-570. [PubMed: 15999095]

5. Cyster JG. Chemokines, sphingosine-1-phosphate, and cell migration in secondary lymphoid organs. Annu Rev Immunol 2005;23:127-159. [PubMed: 15771568]

6. Bokoch GM, Katada T, Northup JK, Ui M, Gilman AG. Purification and properties of the inhibitory guanine nucleotide-binding regulatory component of adenylate cyclase. J Biol Chem 1984;259:35603567. [PubMed: 6323429]

7. Akbar H, Cancelas J, Williams DA, Zheng J, Zheng Y. Rational design and applications of a Rac GTPase-specific small molecule inhibitor. Methods Enzymol 2006;406:554-565. [PubMed: 16472687]

8. Spiegel S, English D, Milstien S. Sphingosine 1-phosphate signaling: providing cells with a sense of direction. Trends Cell Biol 2002;12:236-242. [PubMed: 12062172]

9. Takuwa Y. Subtype-specific differential regulation of Rho family $\mathrm{G}$ proteins and cell migration by the Edg family sphingosine-1-phosphate receptors. Biochim Biophys Acta 2002;1582:112-120. [PubMed: 12069818]

10. Mazo IB, et al. Hematopoietic progenitor cell rolling in bone marrow microvessels: parallel contributions by endothelial selectins and vascular cell adhesion molecule 1. J Exp Med 1998;188:465-474. [PubMed: 9687524]

11. Mazo IB, et al. Bone marrow is a major reservoir and site of recruitment for central memory CD8+ T cells. Immunity 2005;22:259-270. [PubMed: 15723813]

12. Jung $\mathrm{S}$, et al. Analysis of fractalkine receptor $\mathrm{CX}_{3} \mathrm{CR} 1$ function by targeted deletion and green fluorescent protein reporter gene insertion. Mol Cell Biol 2000;20:4106-4114. [PubMed: 10805752]

13. Niess JH, et al. $\mathrm{CX}_{3} \mathrm{CR} 1$-mediated dendritic cell access to the intestinal lumen and bacterial clearance. Science 2005;307:254-258. [PubMed: 15653504]

14. Burnett $\mathrm{SH}$, et al. Conditional macrophage ablation in transgenic mice expressing a Fas-based suicide gene. J Leukoc Biol 2004;75:612-623. [PubMed: 14726498]

15. Sanna MG, et al. Sphingosine 1-phosphate (S1P) receptor subtypes $\mathrm{S}_{1} \mathrm{P}_{1}$ and $\mathrm{S}_{1} \mathrm{P}_{3}$, respectively, regulate lymphocyte recirculation and heart rate. J Biol Chem 2004;279:13839-13848. [PubMed: 14732717]

16. Wei SH, et al. Sphingosine 1-phosphate type 1 receptor agonism inhibits transendothelial migration of medullary T cells to lymphatic sinuses. Nat Immunol 2005;6:1228-1235. [PubMed: 16273098]

17. Liu Y, et al. Edg-1, the G protein-coupled receptor for sphingosine-1-phoshate, is essential for vascular maturation. J Clin Invest 2000;106:951-961. [PubMed: 11032855]

18. Allende ML, et al. G-protein-coupled receptor $\mathrm{S}_{1} \mathrm{P}_{1}$ acts within endothelial cells to regulate vascular maturation. Blood 2003;102:3665-3667. [PubMed: 12869509]

19. Ferron M, Vacher J. Targeted expression of Cre recombinase in macrophages and osteoclasts in transgenic mice. Genesis 2005;41:138-145. [PubMed: 15754380]

20. Filgueira L. Fluorescence-based staining for tartrate-resistant acidic phosphatase (TRAP) in osteoclasts combined with other fluorescent dyes and protocols. J Histochem Cytochem 2004;52:411-414. [PubMed: 14966208]

21. Stoller P, Reiser KM, Celliers PM, Rubenchik AM. Polarization-modulated second harmonic generation in collagen. Biophys J 2002;82:3330-3342. [PubMed: 12023255] 
22. Yu X, Huang Y, Collin-Osdoby P, Osdoby P. Stromal cell-derived factor-1 (SDF-1) recruits osteoclast precursors by inducing chemotaxis, matrix metalloprotease-9 (MMP-9) activity, and collagen transmigration. J Bone Miner Res 2003;18:1404-1418. [PubMed: 12929930]

23. Katayama Y, Battista M, Kao WM, Hidalgo A, Peired AJ, Thomas SA, Frenette PS. Signals from the sympathetic nervous system regulate hematopoietic stem cell egress from bone marrow. Cell 2006;124:407-421. [PubMed: 16439213]

24. Matloubian M, et al. Lymphocyte egress from thymus and peripheral lymphoid organs is dependent on S1P receptor 1. Nature 2004;427:355-360. [PubMed: 14737169]

25. Cyster JG. Chemokines, sphingosine-1-phosphate, and cell migration in secondary lymphoid organs. Annu Rev Immunol 2005;23:127-159. [PubMed: 15771568]

26. Kappos L, et al. Oral fingolimod (FTY720) for relapsing multiple sclerosis. N Engl J Med 2006;355:1124-1140. [PubMed: 16971719]

27. Tedesco-Silva H, et al. FTY720 versus mycophenolate mofetil in de novo renal transplantation: sixmonth results of a double-blind study. Transplantation 2007;84:885-892. [PubMed: 17984842]

28. Kobayashi K, et al. Tumor necrosis factor $\alpha$ stimulates osteoclast differentiation by a mechanism independent of the ODF/RANKL-RANK interaction. J Exp Med 2000;191:275-286. [PubMed: 10637272]

29. Ishii M, et al. RANKL-induced expression of tetraspanin CD9 in lipid raft membrane microdomain is essential for cell fusion during osteoclastogenesis. J Bone Miner Res 2006;21:965-976. [PubMed: 16753027]

30. Okamoto $\mathrm{H}$, et al. Inhibitory regulation of Rac activation, membrane ruffling, and cell migration by the G protein-coupled sphingosine-1-phosphate receptor EDG5 but not EDG1 or EDG3. Mol Cell Biol 2000;20:9247-9261. [PubMed: 11094076] 
a

b $\mathbf{f}$

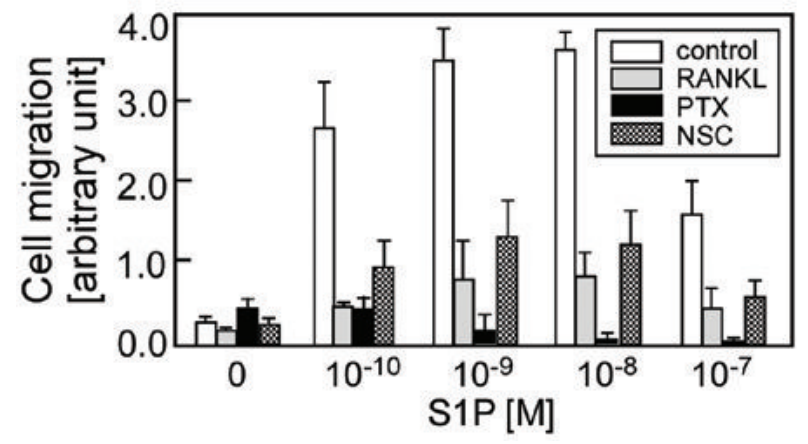

C

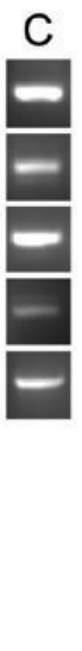

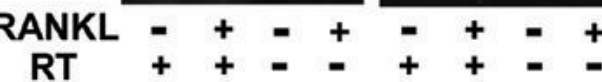

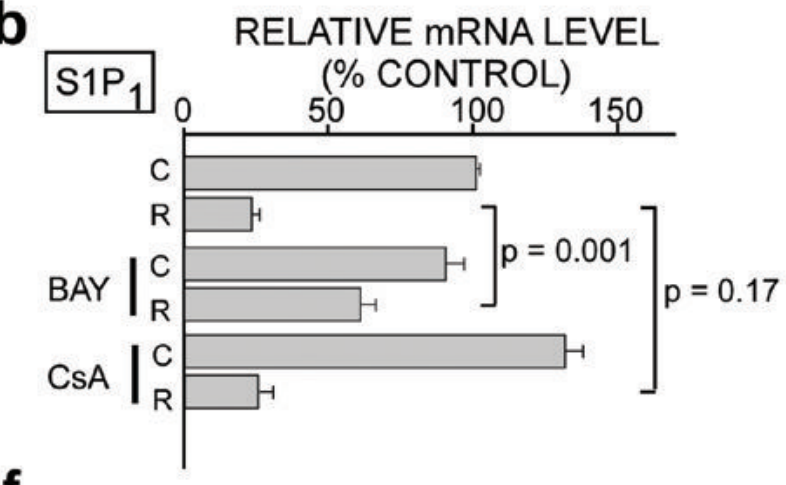

g
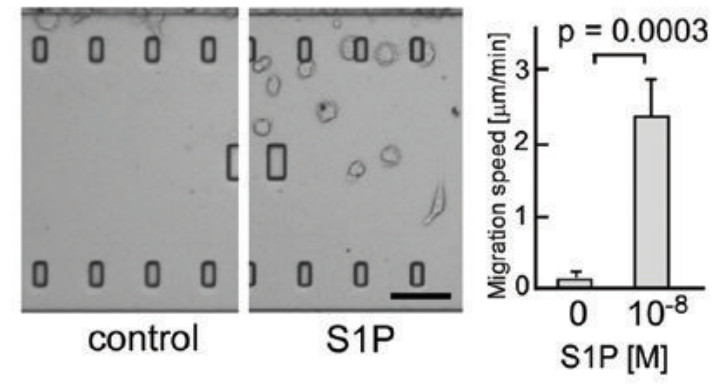

Figure 1. Expression and function of S1P receptors in osteoclast precursor monocytes a, Expression of mRNAs encoding 5 mammalian $\mathrm{S} 1 \mathrm{P}$ receptors $\left(\mathrm{S}_{1} \mathrm{P}_{1}\right.$ to $\left.\mathrm{S}_{1} \mathrm{P}_{5}\right)$ in $\mathrm{RAW} 264.7$ monocytes (left panels) and in mouse bone marrow-derived, M-CSF-dependent monocytes (BM-MDM) (middle panels), detected by RT-PCR. mRNAs for integrin $\alpha_{\mathrm{v}}$ and GAPDH were also analyzed as controls. Total cDNA isolated from mouse thymus was used for positive controls (C, right panels). RT, reverse transcription. b, Quantitative real-time RT-PCR analysis of $\mathrm{S}_{1} \mathrm{P}_{1}$ mRNA expressed in RAW264.7 cells cultured in the absence $(\mathrm{C})$ or presence $(\mathrm{R})$ of RANKL. Cells were treated with BAY11-7085 (BAY; $10 \mu \mathrm{M})$ or cyclosporine A (CyA; 1 $\mu \mathrm{M})$. Error bars represent $\pm \mathrm{SEM}$. c, Immunofluorescent detection of $\mathrm{S}_{1} \mathrm{P}_{1}$ protein (green) in RAW264.7 cells cultured in the absence (left panel) or presence (right panel) of $50 \mathrm{ng} / \mathrm{ml}$ 
RANKL. Nuclei were visualized with propidium iodide (red). Scale bars represent $20 \mu \mathrm{m}$. d, Immunohistochemical analysis of $\mathrm{S}_{1} \mathrm{P}_{1}$ in mouse femoral bone tissues at low (upper) and high (lower) magnification. Staining for $\mathrm{S}_{1} \mathrm{P}_{1}$ (green) (left two panels); merged image with staining for CD9 (red) and transmission (Nomarski image) (right two panels). Arrowheads represent $\mathrm{S} 1 \mathrm{P}_{1}{ }^{\text {high }}$ mononuclear cells adjacent to bone trabeculae (asterisk). Scale bars represent $20 \mu \mathrm{m}$. e, S1P-induced Rac stimulation. RAW264.7 cells were treated with various concentrations of S1P for $15 \mathrm{~min}$ and then analysed for GTP-Rac. f, In vitro chemotactic response of RAW264.7 to S1P gradient. Error bars represent SEM $(\mathrm{n}=6)$. g, In vitro S1P-directed chemotaxis of RAW264.7 dynamically visualized using EZ-Taxiscan ${ }^{\mathrm{TM}}$. Cells were loaded onto the upper chamber and the lower chamber was filled either with normal medium (right panel;

Supplementary Video 1) or with medium containing $10^{-8}$ M S1P (middle panel; Supplementary Video 2). Mean migration speed is shown in right panel. Error bars represent SEM $(n=8)$.

Scale bar represents $100 \mu \mathrm{m}$. 
a
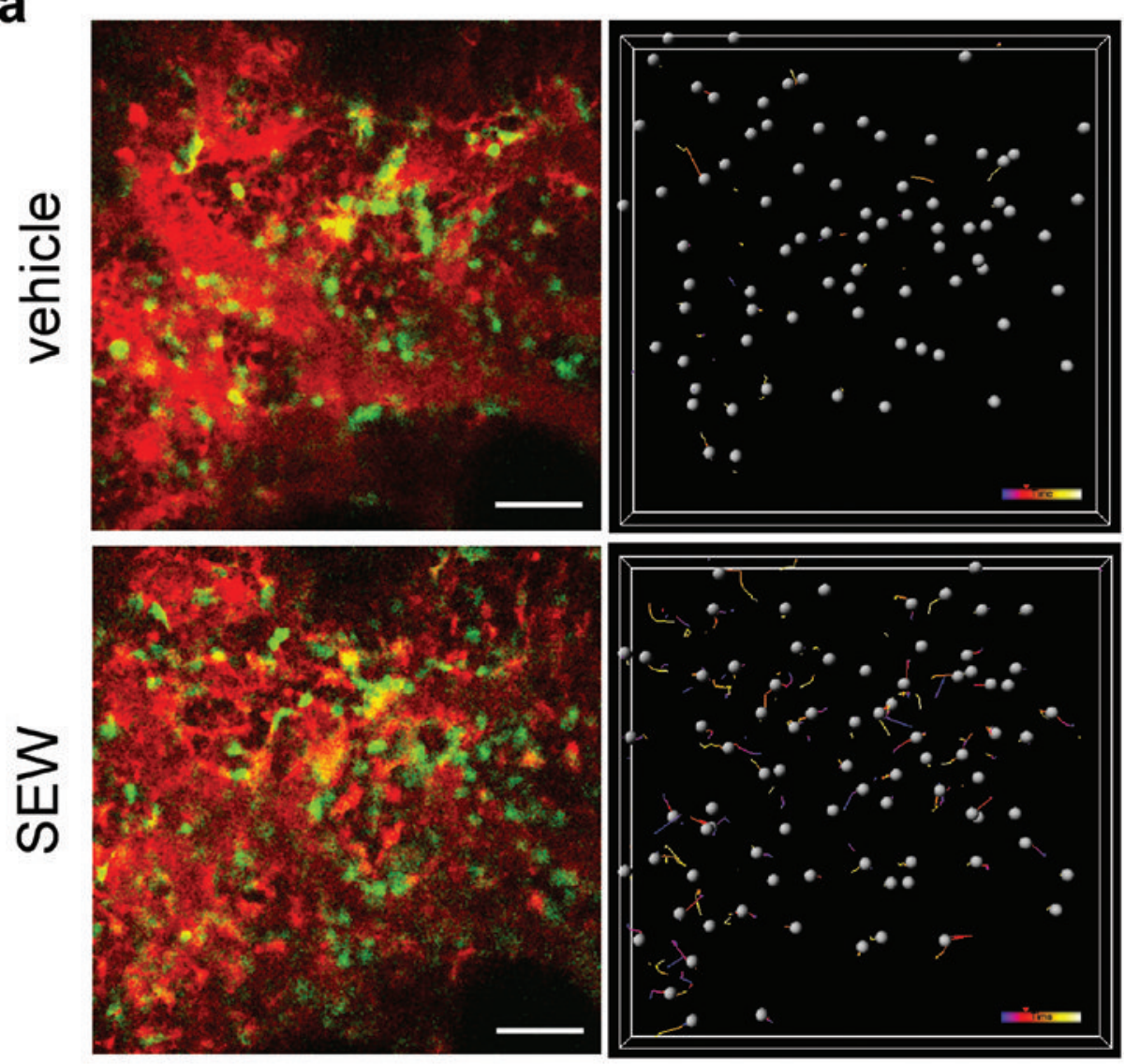

b

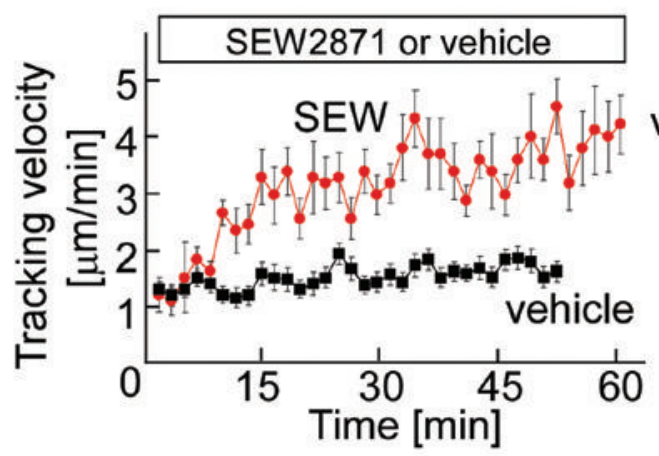

C

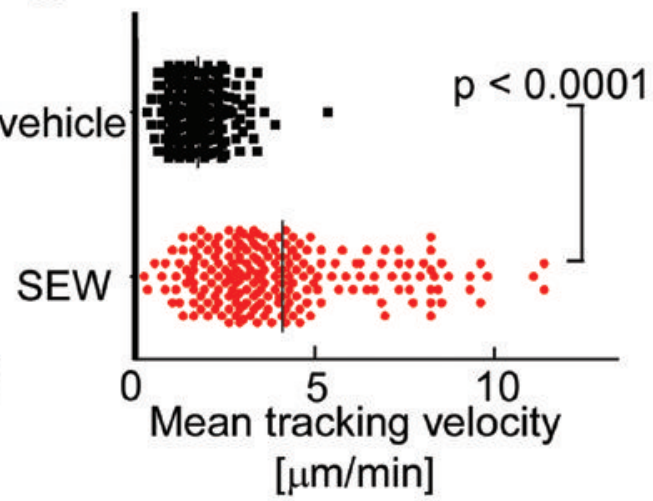

Figure 2. In vivo S1P-mediated increase in motility of osteoclast precursor monocytes visualized using intravital two-photon imaging

a, Intravital two- photon imaging of mouse skull bone tissues of heterozygous $\mathrm{CX}_{3} \mathrm{CR} 1$-EGFP knock-in mice, in the absence (upper panels; Supplementary Video 3) or presence (lower panels; Supplementary Video 4) of the $\mathrm{S}_{1} \mathrm{P}_{1}$ agonist SEW2871 (5 mg/kg). CX ${ }_{3} \mathrm{CR} 1-\mathrm{EGFP}$ positive cells appear green. The microvasculature was visualized by intravenous injection of $70 \mathrm{kDa}$ dextran-conjugated Texas Red (red) (left panels). The movements of $\mathrm{CX}_{3} \mathrm{CR} 1$-EGFP positive cells were tracked for 10 minutes (right panels). Gray spheres represent cells and colored lines show the associated trajectories. Scale bars represent $50 \mu \mathrm{m}$. b, Quantification of $\mathrm{CX}_{3} \mathrm{CR} 1-\mathrm{EGFP}$ positive cell velocity. Tracking velocity over time after application of 
SEW2871 (red circle) or vehicle only (black square) are shown. Data points represent mean values $( \pm$ SEM) of cell velocities in the field at certain time points $(n=15$ for vehicle and $n=$ 14 for SEW 2871). c, Summary of mean velocity of $\mathrm{CX}_{3}$ CR1-EGFP positive cells treated with SEW2871 (red circle) or vehicle (black circle). Data points ( $n=231$ for vehicle and $n=210$ for SEW2871) represent individual cells compiled from 5 independent experiments. 
a control
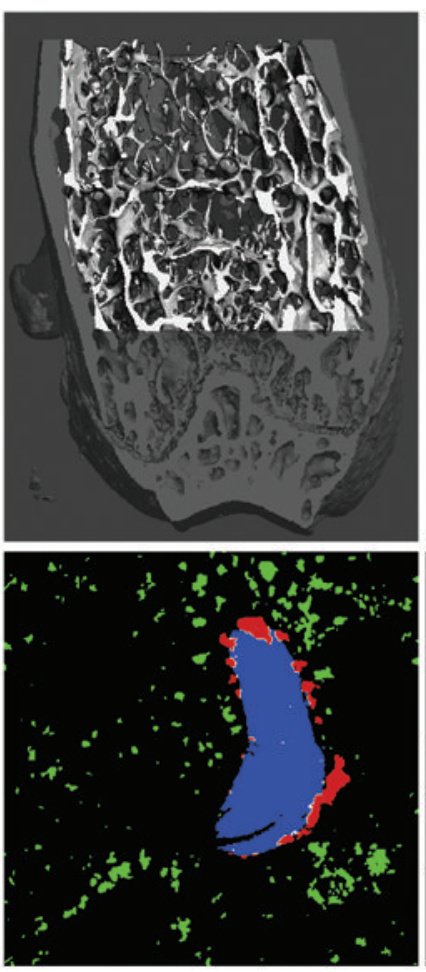

$\mathrm{cS}_{1 \mathrm{P}} \mathbf{1}^{-/-}$
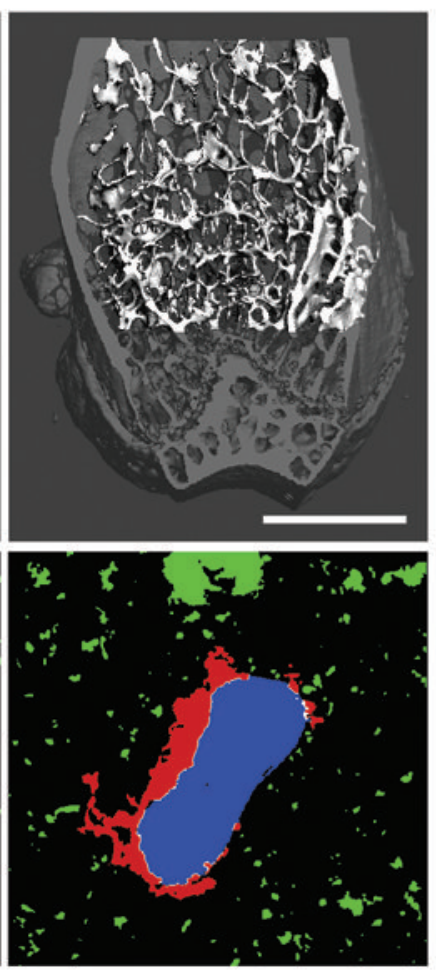

b

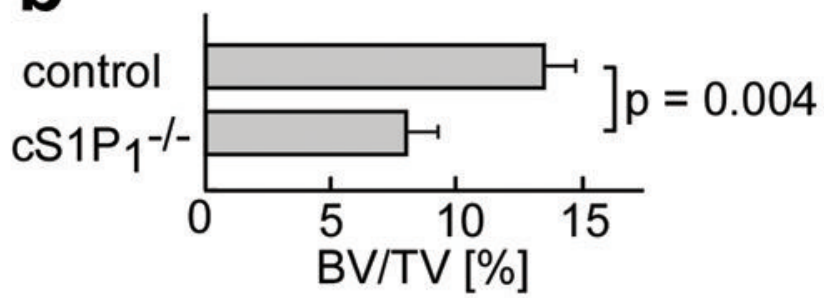

C

Tb.Th. [mm]

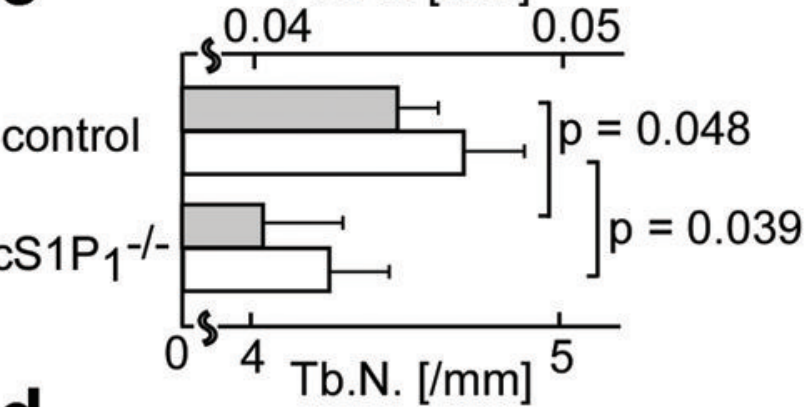

d

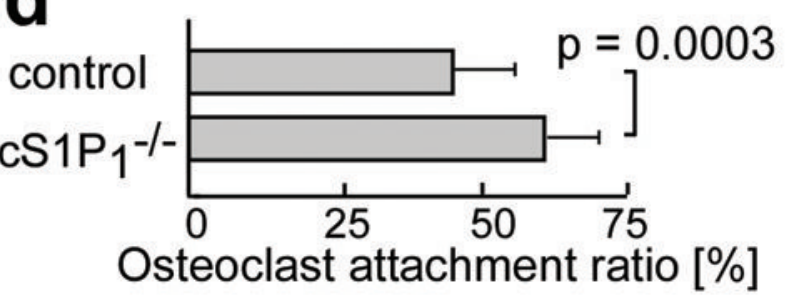

Figure 3. In vivo impact of $\mathrm{S}_{1} \mathrm{P}_{1}$ on bone mineral metabolism

a, Morphohistometric analyses of control and $\mathrm{CS}_{1} \mathrm{P}_{1}^{-/-}\left(\mathrm{S}_{1} \mathrm{P}_{1}\right.$ loxP/loxP $\left.\mathrm{CD} 11 \mathrm{~b}-\mathrm{Cre}\right)$ littermates. Femoral bone samples were analyzed by cone-beam $\mu \mathrm{CT}$ (upper panels) and by histological examination combined with computational quantification for measuring the osteoclast attachment ratio to the bone surface (lower panels) (also see Supplementary Fig. 6 and supplementary material). Blue areas represent bone trabeculae (2nd harmonic fluorescence signal), red and green areas show TRAP-positive osteoclasts that are attached to or detached from bone trabeculae, respectively, and white shows the area of OC/bone attachment. Scale bar (in upper panel) represents $1 \mathrm{~mm}$. b-d, Summary of the data of bone matrix density (bone volume / total volume $=\mathrm{BV} / \mathrm{TV})(\mathbf{b})$, trabecular thickness $(\mathrm{Tb}$.Th.) $(\mathbf{c}$, filled bars $)$ and trabecular density (Tb.N.) (c, open bars) calculated from $\mu \mathrm{CT}$ images, and osteoclast attachment ratio calculated by computational segmentation analyses (d). Error bars represent SEM. $n=3(\mathbf{b}, \mathbf{c})$ and $n=40$ (from 3 mice) (d) for each. 
a
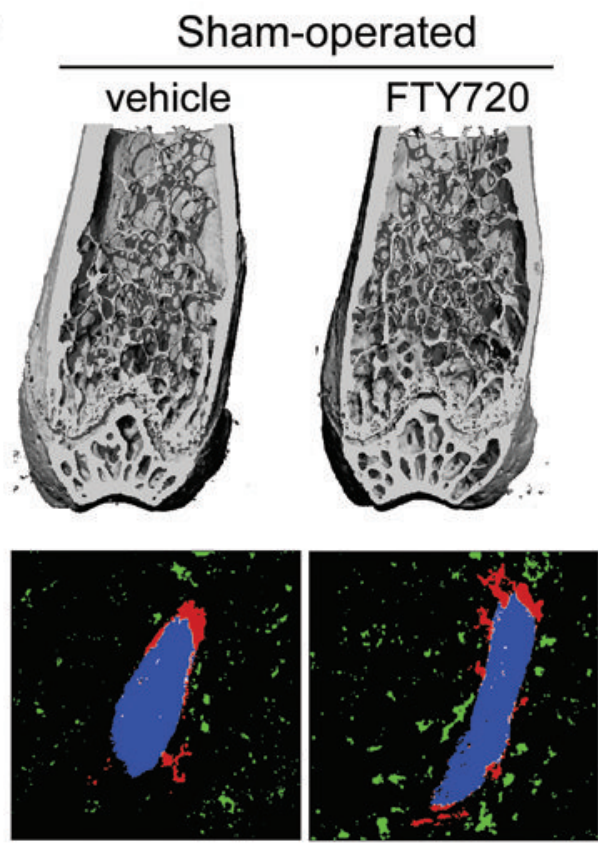

b

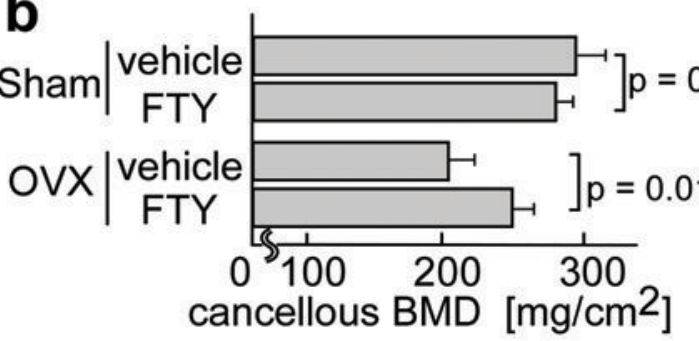

C

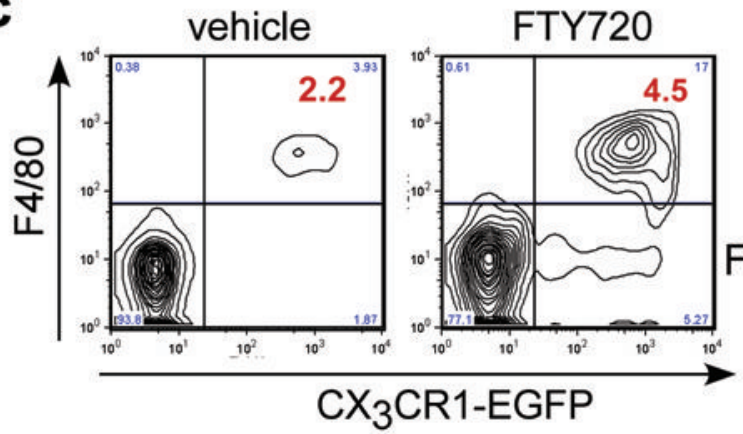

\section{Ovariectomized}
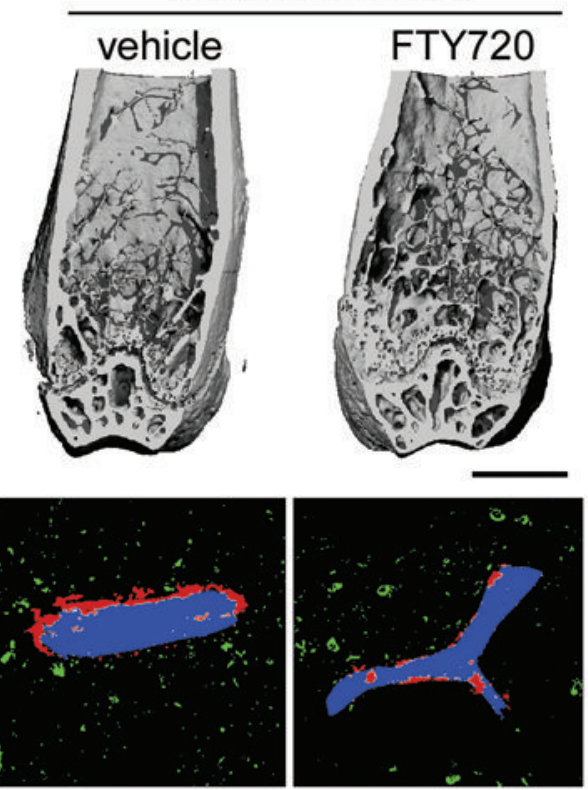
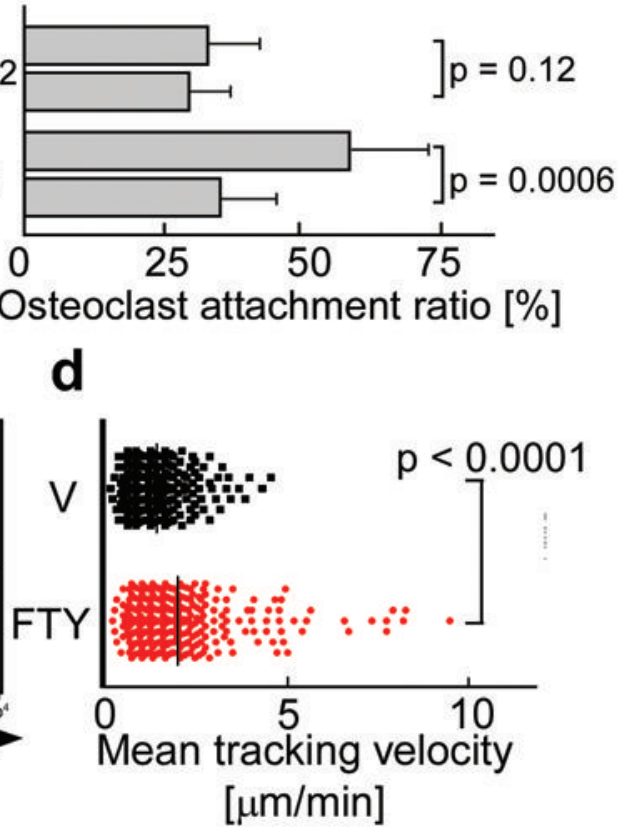

Figure 4. Preventive effect of FTY720 on ovariectomy-induced osteoporosis

a, Effect of FTY720 on bone mineral metabolism. Femurs were collected from mice after four different treatments; sham-operated / vehicle treated, sham-operated / FTY720 treated, ovariectomized / vehicle treated and ovariectomized / FTY720 treated. Bone samples were analyzed by cone-beam $\mu \mathrm{CT}$ (upper panels) and by histological examination combined with computational quantification for measuring the osteoclast attachment ratio to the bone surface (lower panels) (see Supplementary Fig. 5). Scale bar (upper panel) represents $1 \mathrm{~mm}$. b, Summary of the data of cancellous bone mineral density calculated from $\mu$ CT images (left panel) and of osteoclast attachment ratio (right panel). Error bars represent SEM. n = 3 (left panel) and $n=20$ (from 3 mice) (right panel) for each. c, Effect of FTY720 on the composition 
of peripheral mononuclear cells (PMC). PMC collected from $\mathrm{CX}_{3} \mathrm{CR} 1$-EGFP knock-in mice administered vehicle or FTY720 were stained with anti-F4/80 (Alexa647). Absolute numbers of $\mathrm{CX}_{3} \mathrm{CR} 1-\mathrm{EGFP}^{+} \mathrm{F} 4 / 80^{+}$cells $\left[/ 10^{5} \mathrm{ml}\right.$ of peripheral blood] are described in the panels. $\mathbf{d}$, Effect of FTY720 on the mobility of $\mathrm{CX}_{3}$ CR1-EGFP marked cells. Summary of mean tracking velocity of $\mathrm{CX}_{3}$ CR1-EGFP positive cells treated with FTY720 (red circle) or vehicle (black circle). Data points $(n=246$ for vehicle and $n=339$ for FTY720) represent individual cells compiled from 4 independent experiments. Intravital two-photon images of mouse skull bone tissues of heterozygous $\mathrm{CX}_{3}$ CR1-EGFP knock-in mice treated with FTY720 are shown in Supplementary Video 7. 